\title{
NATURE OF SELF-DIFFUSION IN FLUIDS
}

\begin{abstract}
The nature of the self-diffusion in low-molecular fluids is discussed. The particular attention is paid to atomic fluids (such as argon), liquid metals, and associated fluids (such as water). The self-diffusion coefficient in the fluids of all indicated types is considered to be the sum of two components: one of them is associated with the transfer of molecules by hydrodynamic vortex modes, and the other is generated by the circulatory motion of local molecular groups. The both components have a collective nature, they are genetically related to each other and differ only by their scales: the former is mesoscopic, the latter is nanoscopic. Manifestations of the collective vortical transport of molecules as specific features in the time dependence of the root-mean-square displacement of molecules are discussed. Sound arguments are proposed concerning the inadequacy of the activation mechanism of thermal molecular motion in lowmolecular liquids. The immanent contradiction of exponential temperature dependences for the viscosity and self-diffusion coefficients is proved. In all cases, the preference is given to qualitative arguments.
\end{abstract}

Ключові слова: self-diffusion coefficient, shear viscosity, molecular liquids.

\section{Introduction}

Self-diffusion of atoms or molecules in fluids belongs to the simplest kinetic processes. It has been studied in numerous theoretical and experimental works (see, e.g., works $[1,2])$. However, a lot of aspects of the selfdiffusion still remain not quite clear. After the works by I.Z. Fisher [3,4] and L.A. Bulavin [2,5,6], it became clear that the self-diffusion coefficient of fluids is the sum of the collective and, as it was coined in the cited works, "one-particle" components:

$D_{s}=D_{c}+D_{r}$

where $D_{r}$ describes the stochastic motion of molecules on molecular scales.

The emergence of the collective component notion did not invoke principal objections, but the calculation of its magnitude and temperature dependence became associated with serious difficulties due to the lack of reliable data concerning the behavior of the Maxwell relaxation time (MRT). As a rule, this parameter is determined by computer simulations. However, various additional approximations have a too strong effect on the final result. In particular, most of the obtained MRT values do not

(C) M.P. MALOMUZH, 2018

1076 satisfy the inequality [7]

$\tau_{\mathrm{M}}>\nu / c_{l}^{2}$,

where $\nu$ is the kinematic shear viscosity, and $c_{l}$ the longitudinal sound velocity. In essence, this inequality follows from the MRT definition, $\tau_{\mathrm{M}}=\nu / c_{t}^{2}$, and the explicit inequality $c_{t}<c_{l}$, where $c_{t}$ is the high-frequency transverse sound velocity in fluids. As a result, the estimates of the collective component turned out overestimated $[2,8]$. Moreover, the applicability region of the obtained MRT values has not been analyzed, so that the MRT continues to exist even in a vicinity of the critical point, where system's density approaches the values typical of the gaseous state.

There are even more difficulties with the physical interpretation of the so-called "one-particle" component of the self-diffusion coefficient. Let us emphasize from the very beginning that the concept of "one-particle character" is applicable only in the case of rarefied gas, where the motion of a particle during its free-run time is determined only by the corresponding initial parameters. In a dense liquid system, every molecule interacts with all its nearest neighbors, i.e. its motion is also collective. The only difference consists in that no hydrodynamic methods can be used for its description. Attempts to estimate

ISSN 2071-0194. Укр. біз. журн. 2018. T. 63, № 12 
$D_{r}$ on the basis of concepts used in solid state physics are also incorrect. In particular, the inapplicability of the activation mechanism has been repeatedly noted in the literature.

This work is mainly devoted to the discussion of qualitative issues associated with the self-diffusion process in fluids of various types, first of all, in argon and water. It will be shown that 1) the fundamental basis of the collective molecular drift in fluids is the fluidity of the latter; 2) a characteristic indicator of the collective transfer in fluids is a square-root contribution to the root-mean-square displacement of a molecule; 3 ) the so-called "one-particle" contributions to the self-diffusion coefficient are formed by circular (vortical) molecular motions; and 4) the activation mechanism of thermal molecular motion in fluids is inconsistent with the character of this motion.

\section{Hydrodynamic Mechanism of Collective Transfer in Fluids}

In this section, the main attention is focused on peculiarities of the collective molecular drift, which is the most important attribute of the thermal motion in fluids.

\subsection{Collective transfer in fluids}

The most important difference between fluids and solids, which are close by density, is the fluidity of the former. This characteristic is directly associated with the transfer of matter, momentum, heat, and so forth. This transfer is reversible and is not connected with the formation of the self-diffusion, viscosity, thermal conductivity, and so on coefficients. However, the fluidity of fluids is a prerequisite for the formation of fluctuating hydrodynamic fields in them. The space-time evolution of those fields is irreversible and favors the appearance of corresponding contributions to kinetic coefficients.

The formation of collective contributions to the self-diffusion coefficient of fluids is the simplest manifestation of fluctuating hydrodynamic flows that are permanently generated in the system. In essence, the emergence of a collective component in the selfdiffusion coefficient of molecules is as natural and universal as the fluidity of fluids. Chaotic molecular displacements observed on the molecular scale take place independently of those induced by fluctuating hydrodynamic flows, whose scale considerably exceeds molecular sizes. Accordingly, the coefficient of molecular self-diffusion is represented in the form

$D_{s}=D_{c}+D_{r}$

Hence, the main issue is reduced to the following question: What is the share of the collective contribution to the experimental self-diffusion coefficient?

It is worth noting here that a stochastic collective drift of a molecule is very similar, in many aspects, to the random motion of a Brownian particle, which is transferred by a fluctuating hydrodynamic flow like a bobber on rapids of a river. The self-diffusion coefficient $D_{s}$ of Brownian particles is well studied and is described by the Einstein formula [9]

$D_{s}=\frac{k_{\mathrm{B}} T}{6 \pi \eta r_{p}}$,

where $k_{\mathrm{B}}$ is the Boltzmann constant, $T$ the temperature, $\eta$ the shear viscosity coefficient, and $r_{p}$ the radius of a Brownian particle. An amazing feature of this formula is that it produces a quite satisfactory value for the self-diffusion coefficient of molecules, if $r_{p}$ is substituted by the molecular radius. Furthermore, the temperature dependence of the self-diffusion coefficient is also described by this formula quite satisfactorily.

One should expect the collective component $D_{\mathrm{c}}$ of the molecular self-diffusion coefficient to have a structure similar to Eq. (4):

$D_{c}=\frac{k_{\mathrm{B}} T}{6 \pi \eta r_{L}}$

where $r_{L}$ is the effective radius of a "fluid" particle that drifts in the field of thermal hydrodynamic fluctuations like a Brownian particle. To estimate the value of $r_{L}$, we should analyze the properties of the fluctuating hydrodynamic field of velocities, $\mathbf{u}(\mathbf{r}, t)$, in more details.

\subsection{Role of longitudinal}

and transverse modes of the hydrodynamic field of velocities in the formation of the collective self-diffusion component

In the general case, the velocity field of a liquid medium is the sum of two components [10],

$\mathbf{u}(\mathbf{r}, t)=\mathbf{u}_{s}(\mathbf{r}, t)+\mathbf{u}_{p}((\mathbf{r}), \mathbf{t})$. 


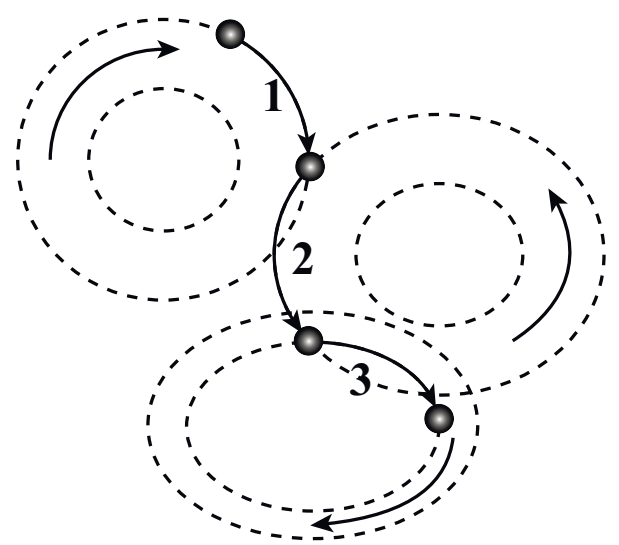

Puc. 1. Schematic diagram of the collective transfer mechanism by fluctuation vortices

The first component describes the medium motions of the vortex type, the second one the motions of the potential type,

$\operatorname{div} \mathbf{u}_{s}(\mathbf{r}, t)=0, \operatorname{rot} \mathbf{u}_{p}(\mathbf{r}, t)=0$.

The potential component of the velocity field is directly associated with the changes of the density and temperature. Those changes are mainly reduced to the translational forward-backward motion of the medium (sound vibrations), which results in negligibly small displacements of the medium particles and, hence, equivalently small contributions to the collective component of the self-diffusion coefficient. On the contrary, vortical displacements lead to a systematic drift of fluid molecules (see Fig. 1).

A molecule in the medium (a circle in Fig. 1) is firstly captured by vortex 1 and transferred over a certain distance. Vortex 1 decays, but there arises another vortex 2 in a vicinity of the molecule and transfers it else over some distance. Subsequent particle displacements occur in this way.

It is very important that the solenoidal (transverse) component of the fluctuating velocity field is described by the diffusion equation

$\frac{\partial \mathbf{u}_{s}(\mathbf{r}, t)}{\partial t}=\nu \Delta \mathbf{u}_{s}(\mathbf{r}, t)$

where $\Delta$ is the Laplace operator, and $\nu$ the kinematic shear viscosity. The same law describes the velocity field of a vortical flow. This fact means that if the hydrodynamic velocity at a certain fixed spatial point located at the distance $r_{0}$ from the vortex center is taken to equal $\mathbf{u}\left(r_{0}, t_{0}\right)=\mathbf{u}_{0}$ at the time moment $t_{0}$, it becomes equal to $\mathbf{u}\left(r_{0}, t\right)=\mathbf{u}_{0}\left(t_{0} / t\right)^{3 / 2}$ at the same point at the time moment $t$. A molecule captured by this vortex moves along a circle of the radius $r_{0}$. Its drift velocity decreases following the same dependence,

$\mathbf{V}_{\mathrm{dr}}(t)=\mathbf{u}_{0}\left(t_{0} / t\right)^{3 / 2}$.

The combination $\varphi_{\mathbf{V}}^{(\mathrm{dr})}\left(t, t_{0}\right)=\left\langle\mathbf{V}_{d r}(t) \mathbf{V}_{d r}\left(t_{0}\right)\right\rangle$, in which the angle brackets denote the averaging over possible values of the initial velocity $\mathbf{u}_{0}$, in accordance with Eq. (9), equals

$\varphi_{\mathbf{V}}^{(\mathrm{dr})}\left(t, t_{0}\right)=\left\langle\mathbf{u}_{0}^{2}\right\rangle\left(\frac{t_{0}}{t}\right)^{3 / 2}$

It is conventionally called the autocorrelation function of the molecular drift velocity.

\subsection{Features of the collective drift of molecules}

Let $\Delta \mathbf{r}_{i}(t)$ denote a displacement of the molecule induced by the velocity field of the $i$-th vortex during the lifetime of the latter: $0<t<t_{V}$. Since different vortices arise statistically independently of one another, the root-mean-square displacement of the molecule, $\Gamma(t)=\left\langle\left(\Delta \mathbf{r}_{i}(t)\right)^{2}\right\rangle$, looks like

$\Gamma(t)=6 D_{V}\left(t-t_{0}\right)$,

where the coefficient $D_{V}$ is determined by the expression

$D_{V}=\frac{1}{6} \frac{\gamma\left(t_{V}\right)}{t_{V}}$

and

$\gamma\left(t_{V}\right)=\left\langle\left(\Delta \mathbf{r}_{i}\left(t_{V}\right)\right)^{2}\right\rangle$

The displacement of the molecule due to the vortex rotation is determined by the standard expression

$\Delta \mathbf{r}_{i}(t)=\int_{t_{0}}^{t} \mathbf{V}_{d r}(u) d u$

The displacement of the molecule during the lifetime of a fluctuation vortex is assumed to be small, so that the influence of the circle curvature can be neglected.

ISSN 2071-0194. Укр. фіз. журн. 2018. T. 63, № 12 
One can directly verify that the root-mean-square displacement of the molecule

$\gamma\left(t_{0}<t<t_{V}\right)=\left\langle(\Delta \mathbf{r}(t))^{2}\right\rangle$

and the autocorrelation function of its drift velocity (10) are coupled by the integral relation

$\gamma(t)=2\left[\left(t-t_{0}\right) \int_{t_{0}}^{t-t_{0}} \varphi_{\mathbf{V}}^{(\mathrm{dr})}(x) d x-\right.$

$\left.-\int_{t_{0}}^{t-t_{0}} \varphi_{\mathbf{V}}^{(\mathrm{dr})}(x) x d x\right]$

where $\varphi_{\mathbf{V}}^{(\mathrm{dr})}(x)=\left\langle\mathbf{u}_{0}^{2}\right\rangle\left(t_{0} / x\right)^{3 / 2}$. The integration leads to the result

$\gamma(t)=6 D_{0}\left(t-t_{0}\right)-12 D_{0} \sqrt{t_{0}\left(t-t_{0}\right)}+C_{0}$,

where

$D_{0}=\frac{2}{3}\left\langle\mathbf{u}_{0}^{2}\right\rangle t_{0}, C_{0}=6 D_{0} t_{0}$

The first term in Eq. (13) has the same structure as the root-mean-square displacement of the molecule during a sufficiently long time interval, as it should be for the self-diffusion process. At the same time, the self-diffusion coefficient depends on the choice of both the initial point inside the vortex (it affects the value of $\left.\left\langle\mathbf{u}_{0}^{2}\right\rangle\right)$ and the initial time moment. This seems to be not quite correct, because the self-diffusion nature should not depend on the choice of $t_{0}$. However, in the framework of our consideration, the choice of the initial moment is not absolutely arbitrary, because it is preceded by the appearance of the vortex in a certain space region at a certain time moment. In the framework of a more rigorous description, the influence of the choice of $t_{0}$ on the self-diffusion coefficient is nullified, and the parameter $t_{0}$ acquires a welldefined physical meaning. Namely, it defines the time moment, when the hydrodynamic description of the corresponding thermal excitation in the fluid becomes possible. A more rigorous description leads to a specific expression for the collective component of the selfdiffusion coefficient.

In our opinion, a more important fact is the presence of the second and third terms in Eq. (13). The appearance of the second term with the quadratic-root dependence on the time is the most important visual indicator of the collective molecular drift within the time interval $t_{0}<t<t_{V}$. The root-meansquare displacement of the molecule becomes nonnegative and approaching the linear law of growth with the time $t-t_{0}$ at

$t>t_{*}, \quad t_{*}=5 t_{0}$.

This result, as well as the appearance of the constant $C_{0}$, is qualitatively consistent with the results of computer simulations of a molecular drift in fluids [2].

\subsection{Qualitative estimation of the collective component of the self-diffusion coefficient}

From a dimensional analysis applied to Eq. (8), it follows that the fluctuating hydrodynamic excitations are characterized by the following hydrodynamic correlation radius:

$r_{C}^{(\mathrm{H})}(t)=\kappa \sqrt{\nu t}$

where $\kappa$ is a proportionality constant. A more detailed analysis of the situation brings about the value $\kappa=2[2]$. From the physical point of view, the most important parameter is the minimum value of the correlation radius. In this case, the hydrodynamic field of velocities in a fluid can be regarded as that created by the motion of an ensemble of fluid, or Lagrangian, particles. The drift velocity of a molecule coincides with the velocity of a liquid particle that includes this molecule. On the other hand, the attention should be paid to that system's responses to external perturbations are different within short and sufficiently long time intervals: in the case of shortterm impacts, the system exhibits elastic properties; otherwise, it demonstrates the fluidity as its basic property. In the case of solenoidal perturbations, it is assumed that those types of system's response are separated by the Maxwell relaxation time $\tau_{\mathrm{M}}$. Accordingly, the minimum size of a Lagrangian particle takes the value

$r_{L}=r_{C}^{(\mathrm{H})}\left(\tau_{\mathrm{M}}\right)=\kappa \sqrt{\nu \tau_{\mathrm{M}}}$.

By its essence, the collective component of the molecular self-diffusion coefficient coincides with the selfdiffusion coefficient of a Brownian particle with radius (16):

$D_{C}=\frac{k_{\mathrm{B}} T}{6 \kappa \pi \sqrt{\nu \tau_{\mathrm{M}}}}$. 


\section{Nature of the Self-Diffusion Component Associated with a Local Intermixing of Neighbor Molecules}

In this section, the arguments concerning the role of activation mechanism in the thermal motion of molecules [11-13] and their self-diffusion in low-molecular fluids are first discussed. Afterward, a new mechanism giving rise to a local intermixing of molecules is

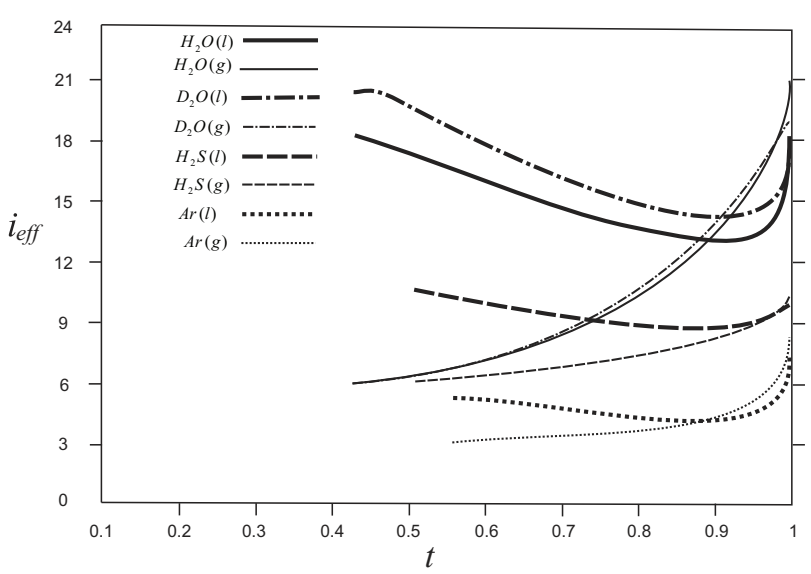

Puc. 2. Temperature dependences of the dimensionless heat capacity $i_{\text {eff }}=\frac{2 c_{V}}{k_{\mathrm{B}} N_{\mathrm{A}}}$ on the coexistence curves of $\mathrm{H}_{2} \mathrm{O}, \mathrm{D}_{2} \mathrm{O}$, $\mathrm{H}_{2} \mathrm{~S}, \mathrm{H}_{2} \mathrm{Se}$, and argon. Experimental data were taken from works $[14,15]$

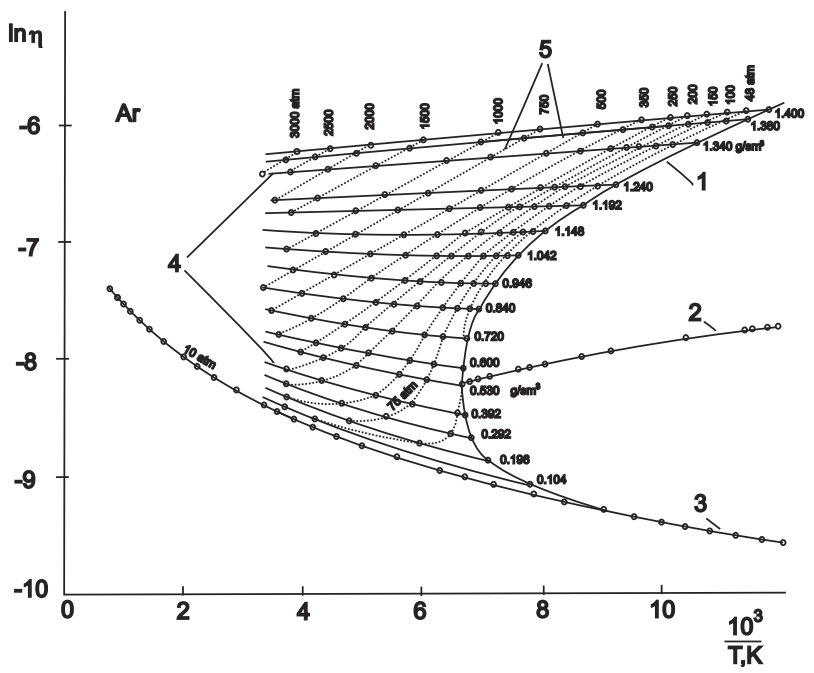

Puc. 3. Dependence of $\ln \eta$ on the reciprocal temperature $1 / T$ for argon: sections 1 and 3 correspond to the liquid and vapor sections of the coexistence curve, section 2 to the viscosity diameter $\eta_{d}(T)=0.5\left(\eta_{L}(T)+\eta_{V}(T)\right)$, section 4 to isochores, and section 5 to isobars. Points correspond to experimental values taken from work [16] considered. For certainty, the consideration is based on the analysis of the temperature dependence of the shear viscosity coefficient, for which the exponential dependence

$\eta=\eta_{0} \exp \left(E_{a} / k_{\mathrm{B}} T\right)$,

where $E_{a}$ is the activation energy, is often used. This behavior corresponds to the following assumption. During the residence time $\tau_{0}$, the liquid molecule vibrates around a certain temporary equilibrium position. Then, within the time interval $\tau_{1} \ll \tau_{0}$, it jumps into a new temporary position with equilibrium vibrations. To make this jump, the molecule has to overcome a certain energy barrier, so that the corresponding kinetic coefficients are proportional to the factor $\exp \left(-E_{a} / k_{\mathrm{B}} T\right)$. This scenario of a molecular thermal motion in fluids is borrowed from the theory of solids with a crystalline or amorphous structure. However, the issue to what extent it corresponds to reality remains unclear. On the example of liquid argon, let us consider below the basic facts that testify to the favor of this scenario of a thermal motion and against it. It will be demonstrated that the thermodynamic properties of liquid argon are really similar, to some extent, to those observed in the crystalline phase. At the same time, it will be shown that the behavior of the simplest transfer coefficients - shear viscosity and selfdiffusion - is definitely inconsistent with the quasicrystalline model of molecular thermal motion.

\subsection{Density and heat capacity of liquid argon}

The density and specific heat of liquid argon are its simplest characteristics. In a vicinity of the argon triple point, they turn out really close to those observed in the crystalline state. In particular, at the triple point itself, the densities of the crystalline and liquid phases differ from each other by no more than $2 \%$. The behavior of the heat capacity of argon, as well as that of water and its homologs, is illustrated in Fig. 2.

With the temperature elevation, the exhibited dependences more and more deviate from the values corresponding to the solid state at the triple point. If a state of the system changes along the coexistence curve up to the critical point, as in Fig. 2, then the density of argon becomes three times lower, and its heat capacity decreases by about $20 \%$. Moreover, at $t=0.9$, the heat capacity becomes even lower than

ISSN 2071-0194. Укр. фіз. журн. 2018. T. 63, № 12 
the heat capacity of argon in the gaseous state. Thus, any similarity between the critical and solid states of argon is out of question.

\subsection{Behavior of shear viscosity}

The behavior of the shear viscosity of liquid argon on its coexistence curve, isochores, and isobars is shown in Fig. 3. Here are arguments that testify to an obvious inconsistency of the features in the presented temperature dependence of the shear viscosity with the quasicrystalline model of molecular thermal motion. For example, the isochoric viscosity values correspond to a dimensionless activation energy $\left(E_{a} / k_{\mathrm{B}} T_{\mathrm{c}}\right)$ that is significantly lower than unity. In other words, the activation energy turns out lower than the thermal noise energy $\left(E_{a}<\right.$ or $\ll$ $\left.\ll k_{\mathrm{B}} T_{\mathrm{c}}\right)$, which has no sense from the physical standpoint. Moreover, the activation energy turns out negative on many isochores. At the same time, the values of the activation energy on the isobars and the coexistence curve seem to be satisfactory (see Table 1).

It should be emphasized that it is the isochoric values of the activation energy that have a direct physical meaning, because, in this case, the macroscopic state of the system changes only owing to the temperature increment. The fulfillment of the condition $E_{a} \gg k_{\mathrm{B}} T$ is mandatory at that.

Let us supplement those results with a simple quantitative analysis of interrelations between the activation energy values typical of various phenomena. We proceed from the expression for the kinematic shear viscosity on isochores, $\left.\nu=\nu_{0} \exp \left(\varepsilon_{a}(n) / T\right)\right)$, where $\varepsilon_{a}(n)=E_{a}(n) / k_{\mathrm{B}}$. A shift along an arbitrary direction in the density-temperature $(n, T)$ plane corresponds to the kinematic shear viscosity variation (see also work [17])

$\frac{d \nu}{d \zeta}=\nu\left[\frac{1}{T} \frac{d \varepsilon_{a}(n)}{d n} \frac{d n}{d \zeta}-\frac{\varepsilon_{a}(n)}{T^{2}} \frac{d T}{d \zeta}\right]$

where $d \zeta$ is a certain linear combination of the density and temperature increments. From Eq. (18), it follows that the effective value of the viscosity activation energy on the isobar equals

$\varepsilon_{\mathrm{eff}}=\varepsilon_{a}(n)-T \frac{d \varepsilon_{a}(n)}{d n} \frac{\beta_{T} n}{d T / d p}$,

where $\beta_{T}$ is the isothermal compressibility. Substituting the numerical valuesof the derivatives into
Таблиця 1. Dimensionless activation

energy values for argon on its isochores, isobars, and coexistence curve

\begin{tabular}{|c|c|r|c|c|}
\hline \multicolumn{5}{|c|}{ Ar } \\
\hline \multicolumn{2}{|c|}{$\rho=$ const } & \multicolumn{2}{c|}{$p=$ const } & $\begin{array}{c}\text { Coexistence } \\
\text { curve }\end{array}$ \\
\hline$\rho / \rho_{c}$ & $E_{a} / k_{\mathrm{B}} T_{\mathrm{c}}$ & $P / P_{c}$ & $E_{a} / k_{\mathrm{B}} T_{\mathrm{c}}$ & $E_{a} / k_{\mathrm{B}} T_{\mathrm{c}}$ \\
\hline 1.12 & -0.67 & 5.14 & 1.37 & 1.75 \\
1.34 & -0.55 & 10.28 & 1.25 & \\
1.49 & -0.46 & 15.42 & 1.17 & \\
1.87 & -0.22 & 20.56 & 1.19 & \\
2.24 & 0.012 & & & \\
2.61 & 0.31 & & & \\
\hline
\end{tabular}

Таблиия 2. Dimensionless activation

energy $E_{a} / k_{\mathrm{B}} T_{m}$ of the self-diffusion coefficient $D_{s}=D_{0} \exp \left(-E_{a} / k_{\mathrm{B}} T\right)$ in the solid and liquid states. $T_{m}$ is the melting temperature

\begin{tabular}{|c|c|c|c|}
\hline Matter & $T_{m}, \mathrm{~K}$ & $E_{a}^{(S)} / k_{\mathrm{B}} T_{m}$ & $E_{a}^{(L)} / k_{\mathrm{B}} T_{m}$ \\
\hline $\mathrm{Ar}$ & 80 & 26.18 & - \\
$\mathrm{Li}$ & 454 & 14.64 & 1.46 \\
$\mathrm{Na}$ & 371 & 13.59 & 2.03 \\
$\mathrm{Rb}$ & 312 & 15.20 & 1.98 \\
$\mathrm{Cs}$ & 301 & 9.56 & 1.92 \\
$\mathrm{Al}$ & 933 & 16.51 & 1.69 \\
$\mathrm{Au}$ & 1337 & 14.86 & 1.58 \\
$\mathrm{Cr}$ & 2130 & 17.46 & - \\
$\mathrm{Pb}$ & 600 & 20.46 & 1.63 \\
$\mathrm{Fe}$ & 1811 & 19.81 & 3.15 \\
$\mathrm{Sn}$ & 505 & 26.22 & 1.39 \\
$\mathrm{Bi}$ & 544.5 & & 1.15 \\
\hline
\end{tabular}

expression (19), we obtain $\varepsilon_{\text {eff }}=1.19$, which is in full agreement with the data in Table 1.

Hence, the effective value of the shear viscosity and self-diffusion activation energy substantially depends on system's state change direction. To obtain its isochoric value, the corresponding recalculation is required. This circumstance was also marked in work $[4,11]$. The values of the ratio $E_{a} / k_{\mathrm{B}} T$ on the argon isochores clearly testify that the character of the thermal motion of its molecules has nothing in common with the quasicrystalline one.

\subsection{On the existence of voids in the structure of a molecular system}

A substantial difference between the self-diffusion coefficients in liquids near their triple point and in 

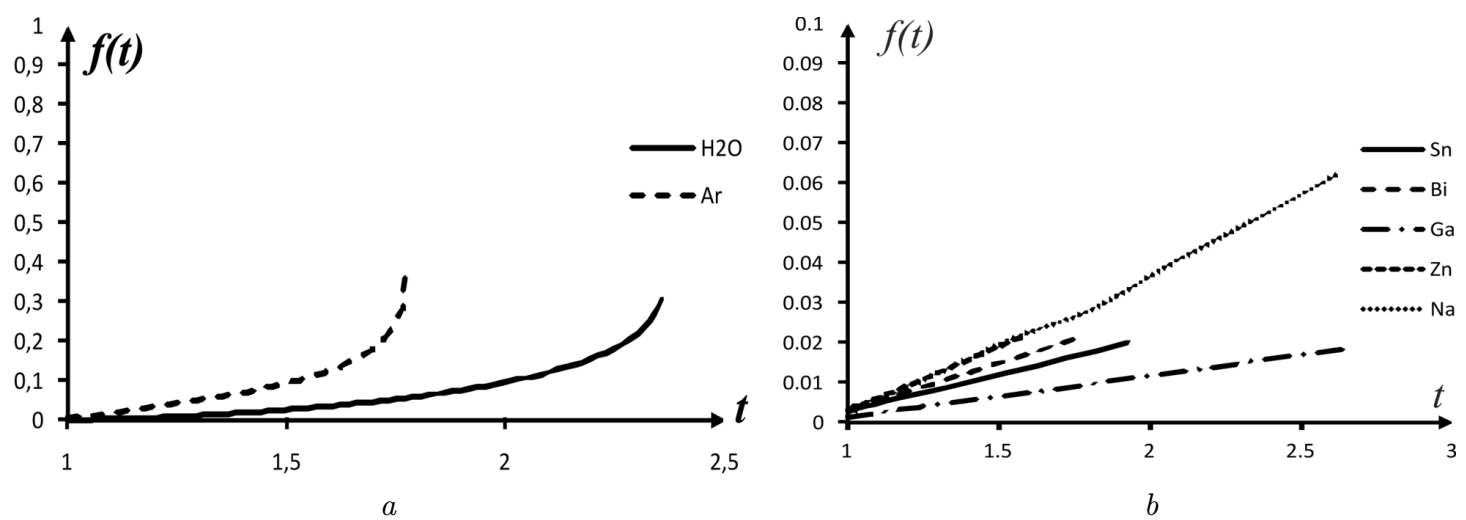

Puc. 4. Temperature dependences $f(t)$ for water, argon, and liquid $\mathrm{Sn}, \mathrm{Bi}, \mathrm{Ga}, \mathrm{Zn}$, and $\mathrm{Na}$

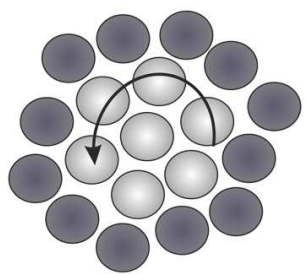

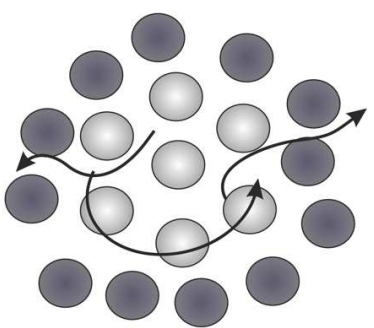

$b$
Puc. 5. Schematic diagram of particle motion in a vicinity of the triple point or melting point $T_{m}(a)$ and near the critical point $T_{\mathrm{C}}(b)$

the corresponding adjacent crystalline states (see Table 2) is often explained by the existence of voids in the liquid state structure [18]. The fallacy of this model follows directly from the temperature dependence of the function $f(t)=\delta / \sigma$, where $\delta=$ $=\left\langle r_{12}\right\rangle-\sigma$ is the average gap between the nearest molecules (ions), $\left\langle r_{12}\right\rangle$ the average distance between the particles, and $\sigma$ the particle diameter. In other words, it describes the relative size of the gap between the nearest neighbor molecules. It is evident that

$f(t)=\frac{v^{1 / 3}(t)-v_{0}^{1 / 3}}{v_{0}^{1 / 3}}$,

where $v$ is the specific volume per particle, and $v_{0}$ the own particle volume, which is quite close to the specific volume at the triple point or the melting point of metals. The temperature dependences $f(t)$ for argon, water, and liquid metals are shown in Fig. 4.

As one can see from Fig. 4, near the triple point of argon and water or the melting points of liquid metals, the relative gap value does not exceed
$1 \%$, i.e. the gap is practically the same as for the system in the crystalline state. In other words, every molecule or ion is in a "cell," whose parameters are close to those in the solid state. As a result, a simple translational motion of molecules in fluids is impossible. At the same time, the values of self-diffusion coefficients in the crystalline and liquid states differ from each other by ten orders of magnitude or more, which is difficult to explain by assuming a fluctuationdriven formation of voids in a vicinity of the diffusing molecule.

\subsection{The self-diffusion component associated with a local intermixing of liquid molecules}

It would be more reasonable, if we address the most important difference between liquids and solids, the fluidity of the former. In other words, the rotation of a group of molecules by a small azimuthal angle is more realistic (Fig. 5). It is important to note that azimuthal motions can be irreversible. In a more general case, the displacements of molecules are combinations of radial and azimuthal displacements of small groups of neighbor molecules. Such permanent small displacements of particles can result in quite large values of the self-diffusion coefficients in fluids. When approaching the critical point, the average distance between the particles grows, and there emerge conditions for a more conventional mechanism of molecular drift, namely, the displacement of molecules into voids that appear in their neighborhood (Fig. 5).

Jump-like (hopping) displacements are also possible, but their contribution to the self-diffusion is expected to be roughly the same as in solids. Such 
a situation takes place, when persons move in a dense crowd (but there are no jumps here!). The corresponding mechanism of self-diffusion in fluids was proposed for the first time in work [21].

To obtain an explicit expression for the component $D_{r}$, which arises due to small rotations of molecular groups, let us apply dimensional reasons and similarity relations. From the former, it follows that $D_{r}$ has to look like

$D_{r} \sim \frac{k_{\mathrm{B}} T}{\eta r_{p}}$.

Hence, the self-diffusion coefficients for molecules with different sizes are related to one another as

$\frac{D_{r}^{(2)}}{D_{r}^{(1)}}=\frac{r_{p}^{(1)}}{r_{p}^{(2)}}$

Taking into account that the self-diffusion coefficients of macromolecules are described by the Einstein formula (4), we conclude that the component $D_{r}$ must have the same form:

$D_{r}=\frac{k_{\mathrm{B}} T}{6 \pi \eta r_{p}}$

where the molecular radius value has to be determined from the analysis of the shear viscosity of a fluid [17].

\subsection{Transfer processes in liquid water}

Properties of water are much more diverse than those of argon. This is a result of the extremely strong interaction and a non-trivial, for low-molecular liquids, angular dependence of the corresponding intermolecular interaction potential. One of the specific features of this interaction is a possibility to extract contributions of hydrogen bonds, which had been considered for a long time as irreducible interaction objects [22].

Actually, the main contribution to the hydrogen bond energy is made by electrostatic forces. There is only a small irreducible component, which is associated with the overlapping of electron shells in neighbor molecules; just this component can be called the hydrogen bond $[23,24]$. Its contribution does not exceed $10-15 \%$ of the total electrostatic interaction energy [24].

Hydrogen bonds, whose interaction energy has an order of magnitude $E_{\mathrm{H}} \sim 5 k_{\mathrm{B}} T_{\mathrm{c}}$, stimulate the

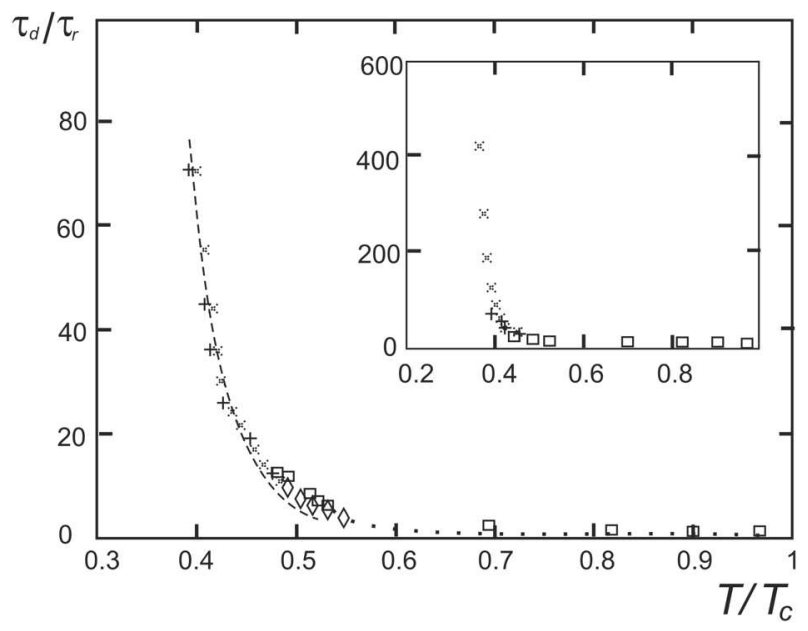

Puc. 6. Temperature dependence of the ratio $\tilde{\tau}_{d}(t)=\tau_{d}(t) / \tau_{r}$. The data were taken from the works: $+[25], \square[29], \times[30]$, and $\diamond[31]$. Points correspond to interpolation values

clustering of various types [25], so that the local structure of water differs significantly from that of argon [26]. The existence of clusters allows the appearance of specific excitations of the vibrational type, which obviously affects the water heat capacity and its temperature dependence (see Fig. 2). It is hydrogen bonds that are responsible for the clustering in liquid water and water vapor, as well as for the considerable differences between the behavior of the heat capacity of water and its homologs, especially $\mathrm{H}_{2} \mathrm{~S}$.

However, some of the thermodynamic and kinetic properties of water are insensitive to clustering effects. The most important among them are the specific volume and the evaporation heat per water molecule. In works $[27,28]$, it was shown that, after the corresponding normalization, the temperature dependences of those parameters on the coexistence curve have the same character as for argon. This nonordinary result can be explained by the fact that water molecules permanently rotate, so that their non-trivial potentials of the intermolecular interaction become self-averaged and acquire a structure that is close to that in argon. In this case, the argonlike behavior of the specific volume and evaporation heat is a logical consequence of the principle of corresponding states.

The specific features of the molecular rotation at various water temperatures are evidently determined by the behavior of the dipole relaxation time $\tau_{d}(t)$ (see Fig. 6). In the figure, $\tau_{r} \sim 2 \pi / \omega_{T}$ is the 
characteristic time of the complete revolution of a molecule, $\omega_{T} \sim \sqrt{k_{\mathrm{B}} T / I}$ a characteristic angular velocity, $I \sim m_{\mathrm{H}} r_{\mathrm{OH}}^{2}$ the moment of inertia of a water molecule, $m_{\mathrm{H}}$ the hydrogen atom mass, and $r_{\mathrm{OH}}$ the distance between the oxygen and hydrogen atoms in a water molecule. As one can see, in the temperature interval $0.6<t<1$, the value of $\tilde{\tau}_{d}(t)$ is close to unity, i.e. the molecular rotation becomes more and more quasifree. Substantial deviations of $\tilde{\tau}_{d}(t)$ from unity are observed only at $t<0.5$ and, especially, in the overcooled region $(t<0.42)$. In this temperature interval, the behavior of $\tilde{\tau}_{d}(t)$ is satisfactorily approximated by the exponential function

$\tilde{\tau}_{d}=\tilde{\tau}_{d}^{(0)} \exp \left(\varepsilon_{\mathrm{H}} / t\right)$,

where $\tau_{d}^{(0)}=5.1 \times 10^{-4}$ and $\varepsilon_{\mathrm{H}}=E_{\mathrm{H}} / k_{\mathrm{B}} T_{\mathrm{c}}=4.71$, which is typical of the activation theory.

Attention is drawn by the activation energy value: it has the same order of magnitude as the dimensionless energy per hydrogen bond. This fact stimulates us to conclude that the rotation of molecules at $t<0.5$ is intermittent, i.e. every rotation by a small angle occurs after only one of hydrogen bonds has been broken.

An important feature of the thermal motion of molecules in water is the applicability of the residence time notion to its description. This quantity, $\tau_{0}$, is directly required, when analyzing the scattering of thermal neutrons $[32,33]$. Since the temperature dependence of $\tau_{0}$ has the same character as $\tilde{\tau}_{d}(t)$, a conclusion can be drawn that a certain configuration of hydrogen bonds remains, in essence, unchanged during the time interval $\tau_{0}$. It is essential that the break of any bond and the formation of a new bond configuration are also connected with a small displacement of the center of mass of a water molecule, $|\Delta \mathbf{r}| \sim 0.1 \AA$, i.e. with a contribution to the self-diffusion process.

In accordance with the aforesaid, we conclude that the temperature dependences of the kinetic coefficients of liquid water should demonstrate different specific features in the following temperature intervals: 1) $T<T_{\mathrm{H}}$, where $T_{\mathrm{H}} \approx 315 \mathrm{~K}[22,32]$; this interval includes both normal and supercooled states; and 2) $T_{\mathrm{H}}<T<T_{\mathrm{c}}$; this interval includes almost all liquid states of water. In the former interval, when the temperature decreases, the character of the thermal motion of water molecules becomes more and more similar to that inherent in hexagonal ice. In the latter interval, when the temperature increases, this character becomes more and more argon-like.

The described features in the thermal motion of water molecules are responsible for the behavior of the temperature dependence of the self-diffusion coefficient. In the temperature interval $T<T_{\mathrm{H}}$, the displacement of a molecule mainly occurs as a result of the cluster decay, and the thermal motion has a quasicrystalline character. In this case, during the transition time $\tau_{1}$, every molecule in the cluster becomes shifted, on average, by a distance equal to a characteristic interparticle distance $\left\langle r_{12}\right\rangle$, which makes it possible to write the following approximate expression for the self-diffusion coefficient in water (see work [34]):

$D_{s} \approx \frac{\left\langle r_{12}\right\rangle^{2}}{6 \tau_{0}} \quad\left(T<T_{\mathrm{H}}\right)$

\subsection{Similarity relation}

In the adjacent temperature interval $T_{\mathrm{H}}<T<T_{\mathrm{c}}$, the magnitude and the character of the temperature dependence of the self-diffusion coefficient in water are assumed to be similar to those in liquid argon (see work [35]):

$D_{s}^{(\mathrm{w})}\left(T_{\mathrm{w}}\right)=\frac{\sigma_{\mathrm{w}}}{\sigma_{\mathrm{Ar}}}\left(\frac{\varepsilon_{\mathrm{w}}}{\varepsilon_{\mathrm{Ar}}} \frac{m_{\mathrm{Ar}}}{m_{\mathrm{w}}}\right)^{1 / 2} D_{S}^{(\mathrm{Ar})}\left(T_{\mathrm{Ar}}\right)$,

$T_{\mathrm{H}}<T<T_{\mathrm{c}}$,

where $T_{\mathrm{w}}$ and $T_{\mathrm{Ar}}$ are the temperatures of the corresponding states for water and argon, respectively,

$T_{\mathrm{w}}=\frac{\varepsilon_{\mathrm{w}}}{\varepsilon_{\mathrm{Ar}}} T_{\mathrm{Ar}}$.

In Eqs. (23) and (24), the following notations are used: $\varepsilon_{\mathrm{Ar}}$ and $\sigma_{\mathrm{Ar}}$ are the Lennard-Jones potential parameters for argon, $\varepsilon_{\mathrm{w}}$ and $\sigma_{\mathrm{w}}$ the parameters of the averaged interaction potential for water molecules similar to the Lennard-Jones potential, and $M_{\mathrm{Ar}}$ and $m_{\mathrm{w}}$ the masses of an argon atom and a water molecule, respectively.

Note that similar relations must be valid for the coefficients of kinematic shear viscosity,

$\nu_{\mathrm{w}}\left(T_{\mathrm{w}}\right)=\frac{\sigma_{\mathrm{w}}}{\sigma_{\mathrm{Ar}}}\left(\frac{\varepsilon_{\mathrm{w}}}{\varepsilon_{\mathrm{Ar}}} \frac{m_{\mathrm{Ar}}}{m_{\mathrm{w}}}\right)^{1 / 2} \nu_{\mathrm{Ar}}\left(T_{\mathrm{Ar}}\right)$,

$T_{\mathrm{H}}<T<T_{\mathrm{c}}$,

ISSN 2071-0194. Укр. біз. журн. 2018. Т. 63, № 12 
and the Maxwell relaxation times,

$\tau_{\mathrm{M}}^{(\mathrm{w})} \frac{\sigma_{\mathrm{w}}}{\sigma_{\mathrm{Ar}}}\left(\frac{\varepsilon_{\mathrm{w}}}{\varepsilon_{\mathrm{Ar}}} \frac{m_{\mathrm{Ar}}}{m_{\mathrm{w}}}\right)^{1 / 2} \tau_{\mathrm{M}}^{(\mathrm{Ar})}$.

From Eqs. (20)-(22), it follows that

$D_{s}^{(\mathrm{w})}(T)=\frac{\nu_{\mathrm{w}}(T)}{\nu_{\mathrm{Ar}}(\lambda T)} D_{s}^{(\mathrm{Ar})}(\lambda T)$,

$\lambda=T_{\mathrm{c}}^{(\mathrm{Ar})} / T_{\mathrm{c}}^{(\mathrm{w})}$,

$T_{\mathrm{H}}<T<T_{\mathrm{c}}$.

Here, we assume that the similarity relations for kinetic and thermodynamic quantities are self-consistent. In particular, for the ratio between the interaction constants and critical temperatures, the following equality is satisfied:

$\frac{\varepsilon_{\mathrm{w}}}{\varepsilon_{\mathrm{Ar}}}=\frac{T_{\mathrm{c}}^{(w)}}{T_{\mathrm{c}}^{(\mathrm{Ar})}}$.

Analogously, from Eqs. (24) and (25), it follows that $r_{L}^{(\mathrm{w})}(T)=\frac{\sigma_{\mathrm{w}}}{\sigma_{\mathrm{Ar}}} r_{L}^{(\mathrm{Ar})}(\lambda T)$

where $r_{L}=2 \sqrt{\nu \tau_{\mathrm{M}}}$ is the appropriate radius of a Lagrangian particle [1]. In this case, for the Lagrange theory of thermal hydrodynamic fluctuations and the method of evaluating, on its basis, the collective component in the self-diffusion coefficient of molecules to be applicable, the inequality

$r_{L}^{(i)}(T) \gg(>) \sigma_{i}, \quad i=\mathrm{Ar}, \mathrm{w}$.

must be satisfied.

The applicability of similarity relations to describe the viscosity and self-diffusion in water was analyzed in work [35] in detail. The analysis demonstrates that those relations lead to a rather good agreement between experimental data and calculation results obtained on their basis for the viscosity and self-diffusion coefficients. This fact allows a conclusion to be made that the character of the thermal motion of water molecules in the whole temperature interval of the liquid water state is the same as in argon, i.e. it has nothing in common with the activation mechanism.

\section{Conclusions}

According to the arguments presented above, a conclusion can be drawn that the molecular thermal motion in fluids and self-diffusion in them have predominantly a non-activation character. The intermixing of particles occurs owing to 1) a collective dri$\mathrm{ft}$ in the field of thermal hydrodynamic fluctuations and 2) irreversible circular motions of small groups of molecules that form closed chains. It is obvious that such circulatory displacements of small molecular groups are genetically associated with vortical hydrodynamic motions giving rise to a collective drift. It is proved that the circulatory particle intermixing is successfully described by the Einstein formula proposed for the description of the self-diffusion of Brownian particles.

When approaching the critical point of the system, the situation chages radically. In this case, the system expands substantially, and the fluctuation-driven formation of voids near the diffusing particle becomes possible (Fig. 2, b). As a result, the kinetic theory can be used to describe the transport processes in enought dense systems [39].

It should be noted that the concept of collective component in the self-diffusion coefficient was introduced for the first time by Oskotskii in work [40] devoted to the analysis of the incoherent scattering of thermal neutrons in water. However, it began to be discussed and used purposefully only after Fisher's works $[3,4]$, where it was independently introduced, and where a method for its determination on the basis of the Lagrange theory of thermal hydrodynamic fluctuations was proposed. Of basic importance were the works by Bulavin and co-authors $[2,5,6]$, in which the value of the collective component in the self-diffusion coefficient was determined for the first time in experiments on the incoherent scattering of thermal neutrons. It was found that the collective component can comprise one fourth and even one third of the total self-diffusion coefficient magnitude. Those works undoubtedly belong to the most profound achievements in molecular physics and the physics of fluids.

Some later, Mikhailenko [41, 42] obtained similar results, by using computer simulations. A further progress and refinement in the Lagrange theory of thermal hydrodynamic fluctuations were made by Lokotosh and the author of this work [43-45]. Computational methods for determining the Maxwell relaxation time of viscous tensions developed by Shakun in works $[7,45]$ are important for estimating the collective component of the self-diffusion coefficient of mo- 


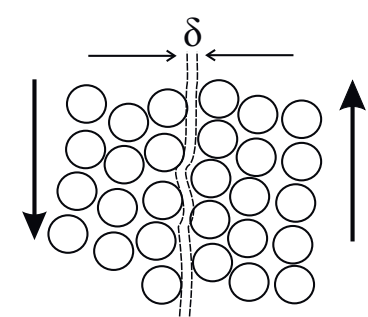

Puc. 7. Illustration of the motion of molecular layers with respect to one another

lecules. In works [46, 47], Malenkov and Naberukhin developed computer methods for the determination of the size of a Lagrangian particle and explicitly visualized vortical flows that play such an important role in the collective transfer [48]. Makhlaichuk's works [4951] played a key role in describing the processes of self-diffusion in simple liquids, liquid metals, water, and aqueous electrolyte solutions. A detailed study of the incoherent scattering of thermal neutrons in water and aqueous solutions was made in the works by Pankratov et al. [31,32].

The described scenario of a thermal motion in fluids also leads to another mechanism of shear viscosity formation. The jump-like motion of molecules from one molecular layer into an adjacent one that moves with respect to the former seems to be hardly probable, because the average gap between the neighbor molecules is much narrower than the molecular diameter:

$2\left(v-v_{0}\right)^{1 / 3} \ll v_{0}^{1 / 3}$.

Accordingly, the shear viscosity of fluids is formed by friction effects between the molecular layers that move with respect to one another (Fig. 7).

A more detailed analysis of the issues discussed above will be made in a separate work.

To summarize, I would like to thank Prof. Leonid Bulavin for his stable interest to all issues raised in this work. Clear formulations of many questions and corresponding answers would be impossible without permanent discussions with T.V.Lokotosh, G.G. Malenkov, V.M. Makhlaichuk, Yu.I. Naberukhin, and K.S. Shakun.

1. E.I. Kharkov, V.I. Lysov, V.E. Fedorov. Physics of Liquid Metals (Vyshcha Shkola, Kyiv, 1979) (in Russian).

2. L.A. Bulavin, T.V. Lokotosh, N.P. Malomuzh. Role of the collective self-diffusion in water and other liquids. J. Mol. Liq. 137, 1 (2008).
3. I.Z. Fisher. Hydrodynamic asymptotics of autocorrelation function of molecular velocity in classical fluid. Zh. Èksp. Teor. Fiz. 61, 1647 (1971) (in Russian).

4. N.P. Malomuzh, I.Z. Fisher. On the collective nature of thermal motion in fluids. Fiz. Zhidk. Sost. 1, 33 (1973) (in Russian).

5. L.A. Bulavin, A.A. Vasilkevich, A.K. Dorosh, V.T. Krotenko, V.I. Slisenko. Self-diffusion of water in aqueous solutions of singly charged electrolytes. Ukr. Fiz. Zh. 31, 1703 (1986) (in Russian).

6. L.A. Bulavin, P.G. Ivanitskii, V.T. Krotenko, V.N. Lyaskovskaya. Neutron studies of water self-diffusion in aqueous electrolyte solutions. Zh. Fiz. Khim. 61, 3270 (1987) (in Russian).

7. N.P. Malomuzh, K.S. Shakun. Maxwell relaxation time for argon. Physica (to be published).

8. T.V. Lokotosh, N.P. Malomuzh, K.N. Pankratov. Thermal motion in water-electrolyte solutions according to quasielastic incoherent neutron scattering data. J. Chem. Eng. Data 55, 2021 (2010).

9. A. Einstein. Eine neue Bestimmung der Molekuldimensionen. Ann. Phys. 19, 289 (1906).

10. E.M. Lifshitz, Fluid Mechanics (Pergamon Press, 1993).

11. J. Frenkel. Kinetic Theory of Liquids (Dover, 1955).

12. E.N. da C. Andrade. The viscosity of liquids. Proc. Phys Soc. 52, 748 (1940).

13. H. Eyring. Viscosity, plasticity, and diffusion as examples of absolute reaction rates. J. Chem. Phys. 4, 283 (1936).

14. CRS Handbook of Chemistry and Physics: A Ready-Reference Book of Chemical and Physical Data. Edited by R.C. West, (CRS Press, 1996).

15. NIST Chemistry WebBook, NIST Standard Reference Database Number 69. Edited by P.J. Linstrom, W.G. Mallar [http://webbook.nist.gov].

16. V.P. Slusar, N.S. Rudenko, V.M. Tretyakov. Experimental study of the viscosity of simple substances on the saturation line and under pressure. II Argon, krypton, xenon. $U k r$. Fiz. Zh. 17, 1257 (1972) (in Russian).

17. V.N. Makhlaichuk. Kinematic shear viscosity of liquid alkaline metals. Ukr. J. Phys. 62, 672, (2017).

18. O.Ya. Samoilov, Structure of Aqueous Electrolyte Solutions and the Hydration of Ions (Consultants Bureau, 1965).

19. C. Jayaram. Temperature dependence of self diffusion in liquid metals [https://archive.org/details/ in.ernet.dli.2015.190768].

20. T. Iida, N. Tripathi, M. Isac, R.I.L. Guthrie. Models and equations for atomic transport coefficients of liquid metals: Viscosity and self-diffusivity. Mater. Sci. Forum 539-543, 2509 (2007).

21. T.V. Lokotosh, M.P. Malomuzh, K.M. Pankratov, K.S. Shakun. New results in the theory of collective selfdiffusion in liquids. Ukr. Fiz. Zh. 60, 697 (2015) (in Ukrainian).

22. G.C. Pimentel, A.L. McClellan. The Hydrogen Bond (Freeman, 1960).

ISSN 2071-0194. Укр. біз. журн. 2018. T. 63, № 12 
23. M.D. Dolgushin, V.M. Pinchuk. Theoretical study of the hydrogen bond nature by comparative calculations. Preprint ITP-76-49G (ITP, Kyiv, 1976) (in Russian).

24. P.V. Makhlaichuk, M.P. Malomuzh, I.V. Zhyganiuk. Nature of hydrogen bond in water. Ukr. J. Phys. 57, 113 (2012).

25. D. Eisenberg, V. Kauzmann. The Structure and Properties of Water (Oxford Univ. Press, 1969).

26. C.A. Croxton. Liquid State Physics - A Statistical Mechanical Introduction (Cambridge Univ. Press, 1974).

27. N.P. Malomuzh, V.P. Oleynik. Nature of the kinematic shear viscosity of water. J. Struct. Chem. 49, 1055 (2008).

28. L.A. Bulavin, A.I. Fisenko, N.P. Malomuz. Surprising properties of the kinematic shear viscosity of water. Chem. Phys. Lett. 453, 183 (2008).

29. K. Okada, M. Yao, Y. Hiejima, H. Kohno, Y. Kojihara. Dielectric relaxation of water and heavy water in the whole fluid phase. J. Chem. Phys. 110, 3026 (1999).

30. H.R. Pruppacher. Self-diffusion coefficient of supercooled water. J. Chem. Phys. 56, 101 (1972).

31. K. Simpson, M. Karr. Diffusion and nuclear spin relaxation in water. Phys. Rev. 111, 1201 (1958)

32. L.A. Bulavin, N.P. Malomuzh, K.N. Pankratov. The character of the thermal motion of water molecules according to the data of quasi-elastic incoherent slow neutron scattering. Zh. Strukt. Khim. 47, 54 (2006) (in Russian).

33. L.A. Bulavin, N.P. Malomuzh, K.N. Pankratov. Specific features of self-diffusion in water.Zh. Strukt. Khim. 47, S54 (2006) (in Russian).

34. L.A. Bulavin, N.P. Malomuzh. Upper temperature limit for the existence of living matter. J. Mol. Liq. 124, 136 (2006).

35. T.V. Lokotosh, S. Magazu, G. Maisano, N.P. Malomuzh. Nature of self-diffusion and viscosity in supercooled liquid water. Phys. Rev. E 62, 3572 (2000).

36. P.V. Makhlaichuk, V.N. Makhlaichuk, N.P. Malomuzh. Nature of the kinematic shear viscosity of low-molecular liquids with averaged potential of Lennard-Jones type. J. Mol. Liq. 225, 577 (2017).

37. N.P. Malomuzh, K.S. Shakun, A.A. Kuznetsova. New possibilities provided by the analysis of the molecular velocity autocorrelation function in liquids. Ukr. Fiz. Zh. 63, 317 (2018) (in Ukrainian).

38. V.M. Makhlaichuk. Qualitative properties of shear viscosity in liquids. Ukr. Fiz. Zh. (to be published) (in Ukrainian).

39. P. Resibois, M. De Leener. Classical Kinetic Theory of Fluids (Wiley, 1978)

40. V.S. Oskotskii. To the theory of quasi-elastic scattering of cold neutrons in liquids. Fiz. Tverd. Tela 5, 1082 (1962) (in Russian).

41. S.A. Mikhailenko, V.V. Yakuba, A.E. Butko. Self-diffusion and nuclear magnetic relaxation in methane-argon liquid mixtures. Fiz. Nizk. Temp. 4, 562 (1978) (in Russian).

42. S.A. Mikhailenko, V.V. Yakuba. Self-diffusion and nuclear magnetic relaxation in liquid propylene and its mixtures with krypton. Ukr. Fiz. Zh. 27, 712 (1982) (in Russian).
43. T.V. Lokotosh, N.P. Malomuzh. Lagrange theory of thermal hydrodynamic fluctuations and collective diffusion in liquids. Physica A 286, 474 (2000).

44. T.V. Lokotosh, N.P. Malomuzh. Manifestation of the collective effects in the rotational motion of molecules in liquids. J. Mol. Liq. 93, 95 (2001).

45. T.V. Lokotosh, N.P. Malomuzh, K.S. Shakun. Nature of oscillations for the autocorrelation functions for transversal and angular velocities of a molecule. J. Mol. Liq. 96-97, 245 (2002).

46. V.P. Voloshin, G.G. Malenkov, Yu.I. Naberukhin. The study of collective motions in computer models of water. Large-scale and long-term correlations. Zh. Strukt. Khim. 54 S2, 239 (2013) (in Russian).

47. G.G. Malenkov, Y.I. Naberukhin, V.P. Voloshin. Collective effects in molecular motions in liquids. Russ. J. Phys. Chem. A 86, 1378 (2012).

48. A.V. Anikeenko, G.G. Malenkov, Yu.I. Naberukhin. Vizualization of the collective vortex-like motions in liquid argon and water: Molecular dynamic simulation. J. Chem. Phys. 148, 094508 (2018).

49. N.P. Malomuzh, V.N. Makhlaichuk. Theory of self-diffusion in liquid metals. Rasplavy 5, 561 (2018) (in Russian).

50. N.P. Malomuzh, V.N. Makhlaychuk. Peculiarities of selfdiffusion and shear viscosity in transition and posttransition metals. Rasplavy 5, 578 (2018) (in Russian).

51. V.M. Makhlaichuk. Shear viscosity of aqueous electrolyte solutions. Ukr. Fiz. Zh. (to be published) (in Ukrainian).

Received 04.11.18.

Translated from Ukrainian by O.I. Voitenko

\section{М.П. Маломуж}

\section{ПРИРОДА САМОДИФУЗІЇ В РІДИНАХ}

$\mathrm{P}$ е $з$ ю м е

Робота присвячена обговоренню природи самодифузіі в низькомолекулярних рідинах. Особлива увага приділяється атомарним рідинам типу аргону, рідким металам та асоційованим рідинам типу води. Підкреслюється, що коефіцієнт самодифузії усіх рідин зазначеного типу є сумою двох складових: однієі, що є зумовленою переносом молекул гідродинамічними вихровими модами, і другої, що породжується циркуляторним рухом локальних груп молекул. Обидві складові мають колективну природу, є генетично пов'язаними й відрізняються між собою тільки масштабами: перші є мезоскопічними, другі - наноскопічними. Обговорюється прояв колективного вихрового переносу молекул у специфіці часової залежності середньоквадратичного зміщення молекули. Подаються вагомі аргументи щодо неадекватності активаційного механізму теплового руху молекул у низькомолекулярних рідинах, доводиться внутрішня суперечливість експоненціальних залежностей для коефіцієнтів в'язкості й самодифузіі. В усіх випадках перевага надається, перш за все, якісним аргументам. 